\title{
Segmentation of Lung Lobes and Fissures for Surgical Pre Planning
}

\author{
S.N. Kumar \\ Assistant Professor, Mar Ephraem College of \\ Engineering and technology, Marthandam
}

\author{
M.Marsaline Beno, \\ Phd, HOD, St.Xaviers Catholic College of \\ Engineering of Engineering, Nagercoil,
}

\begin{abstract}
Medical imaging is the technique that is used to create images of the human body or parts for clinical purposes (medical procedures seeking to reveal diagnose or examine disease). The CT images provide detailed information of anatomy of lungs, which could be used for better surgical planning of treating Lung Cancer This Work, proposes a method for Segmentation of Lung lobes and fissures for surgical planning of treating Lung Cancer. This work presents a 'lobe and fissure' segmentation algorithm that uses a two stage approach. This work presents a threshold based lobe segmentation algorithm. Modified adaptive fissure sweeping and Wavelet Transform are used to segment Lung fissures in $\mathrm{CT}$ images. The unseen or partially seen fissures are enhanced and segmented. The main modules of this work are Preprocessing, Lung Segmentation by Iterative Threshold method, Fissure Region Sweeping, Wavelet Transform, Otsu's binarization, Fissure verification, Interpolation and Extension of Fissures. In the Preprocessing work noises are reduced. The preprocessing is done by fuzzy filter. In the next module lung area are segmented by Iterative Threshold Method. Then the fissure regions are collected. Next Wavelet Transform generates the features. Then Otsu's method is adopted for binarization. Next Interpolation is done and fissure is extended up to needed length. Finally Fissure information are extracted and displayed.
\end{abstract}

\section{INTRODUCTION}

LUNG cancer is the deadliest cancer in world. Lobectomy or surgical removal of the diseased lung lobes is the preferred option for treating lung cancer. On CT, a fissure can have a variety of appearances [1], which makes its detection and segmentation challenging. A Snake is an energy minimizing spine guided by external constraint forces and influenced by image forces that pull it toward features such as line and edges [2].In practice, dilations and erosions are usually employed in pairs either dilation of an image followed by the erosion of the dilated result, or image erosion followed by dilation [3]. The cad system for screening of lung cancer is developed for screening of lung cancer is developed to equal the ability of expert physicians [4][5].There are however no detection algorithms of the pulmonary lobes which are an important part in the detection of the pulmonary configuration in the clinical diagnosis. The segmentation itself is performed on the combined image using an interactive 3D watershed algorithm [6] which allows an iterative refinement of the results. Unfortunately, the thoracic airways are not the only obstacle for a separation of the lungs. Predominantly emphysematous lungs tend to be inflated by the excessive lung pressure originating in the disease. Takahiro [7] estimates the boundary surface between lung lobes by fitting curved surface. The fitting is performed with fuzzy control, and it searches the boundary where the density of tubular tissues is low. Image segmentation based contour models are also there. Snake is an active contour model [8] which is used extensively in computer vision and image processing applications particularly to locate the object the boundaries. An anatomic pulmonary atlas [9] can be used to automatically segment the oblique lobar fissures. A fissure is formed when the visceral pleura of adjacent lobes contact each other with thickness on the order of $0.2 \mathrm{~mm}$ An image transformation called "ridge map" can be used for enhancing the appearance of fissures on CT.

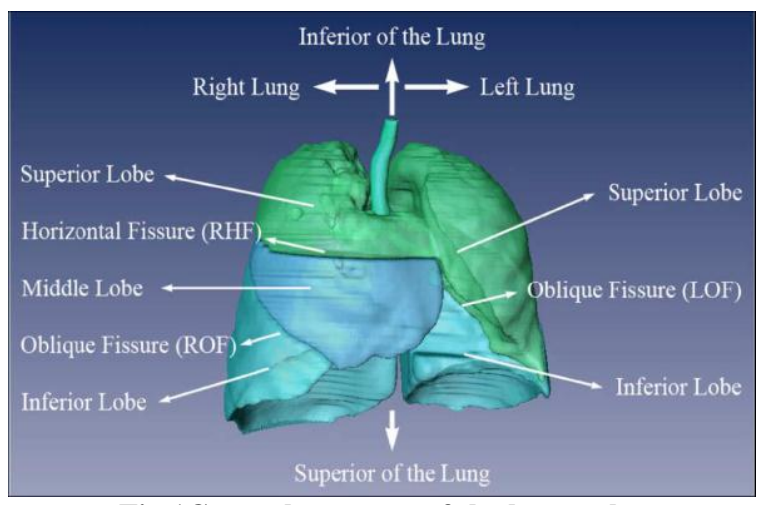

Fig.1General anatomy of the human lungs

A curve-growing process, modeled by a Bayesian network, is described that is influenced by both the features of the ridge map and prior knowledge of the shape of the fissure [10]. The human lungs are composed of distinct anatomic compartments called lobes [11]. Since they represent functional units, the lobes have their own bronchial and vascular systems. These systems are solely connected close to the hilus, which is defined as the area where bronchi as well as the pulmonary arteries and veins enter the lungs. The unique characteristic of this scheme is the representation of fissures in the form of implicit functions using Radial Basis Functions (RBFs) [12], capable of seamlessly interpolating "holes" in the detected fissures and smoothly extrapolating the fissure surfaces.An automatic method for the segmentation and analysis of fissures can be done based on the information provided by the segmentation and analysis of the airway and vascular trees [13].The information is used to provide a close approximation to the fissures using the water shed transform.

\section{PROPOSED METHODOLOGY} 2.1 Preprocessing

Preprocessing is done on the CT image of lung for removal of noise. A fuzzy filter [14] can be used for the removal of noise from CT image. The filter operation involves two levels. In the first level fuzzy derivative is calculated for eight directions. The second level uses fuzzy derivative to perform smoothing along with fuzzification and defuzzification. Both levels are based on fuzzy rules. In particular, the shape of the 
membership functions is adapted in accordance with the remaining noise level after each iteration, making use of the distribution of the homogeneity in the image. The fuzzy filter is found to be best while comparing with other preprocessing filters like wiener filter, median filter, since it is having high PSNR and MSE value.

\subsection{Iterative Thresholding Technique}

In the proposed method iterative thresholding is used. The step by step procedure is follows the first step is the choosing of initial threshold value. Here the initial threshold value is given by $(255 / 2)=128$. The gray level value of each pixel is compared with the initial threshold value in such a way that if the pixel gray value is greater than initial threshold value, the pixel gray value is replaced by 255.If the pixel gray value is less than the initial threshold value, the gray value is replaced by 0 .The next step is to determine the bodyvoxelsum and nonbodyvoxel sum and the bodyvoxel count, nonbodyvoxel count is determined. The bodyvoxel mean and nonbodyvoxel mean is determined. The new threshold value is given by average of mean NonBody Voxel and mean BodyVoxel.With this new threshold value the process is repeated, the no iterations chosen here are 6 .

\subsection{Morphological Operation}

After this extraction, initial thresholding may result in irregular lung boundaries due to lesions and bronchial trees. To remedy this, we applied a circular morphological closing operator to the lungs image. We used a filter size of $10 \times 10$ pixels) to ensure smooth lung boundaries while retaining the original lung shape.

\subsection{Stationary Discrete Wavelet Transform}

Unlike in conventional wavelet transforms, where the resulting coefficient images are down sampled, the stationary DWT uses no down sampling. The resulting approximation and detail coefficient images thus have the same dimensions as the original images. The stationary 2-D DWT yields four sub images containing a low-pass version of the original image and three high-pass filtered images: horizontal, vertical, and diagonal. Among the four images generated from this transform (low-pass, horizontal, vertical, and diagonal), The algorithm uses the horizontal detail image for further processing because most lobar fissures appear horizontally across the fissure

\subsection{Otsu's Method and Fissure Searching}

The lobe segmentation algorithm then uses Otsu's method to create binary images in black and white from the horizontal detail images. Otsu's method is a thresholding technique that automatically chooses a threshold to maximize between the class variances of an image. This method results in binary images, containing continuous or semi continuous lines representing the actual fissures. To identify the actual fissure locations and curvatures, the lobe segmentation algorithm uses a fissure search technique to look for the longest continuous lines crossing the fissure regions, which signifies the actual fissures. The fissure search technique conducts pixel-by-pixel analysis and automatically places anchor points at a distance of 5 pixels apart along the identified fissures.

\subsection{Interpolation and Extension}

The interpolation uses pixels from the two most adjacent correct fissures for estimating the location and curvature of these incorrect fissures. Linear interpolation of the fissure pixels is sufficient to yield a good estimation. Finally, the lobe segmentation algorithm extends fissures that do not extend to vessels or edges of the lungs. The algorithm calculates an average angle for the extension of a fissure by using the last four anchor points of the fissure.

\section{RESULTS}

At first the $\mathrm{Ct}$ image of the lungs is subjected to preprocessing. The preprocessed image is then used for fissure analysis. The efficency of the filter is determined from the PSNR value. This ratio is often used as a quality measurement between the original and preprocessed image. The higher the PSNR, the better the quality of the preprocessed image.The Mean Square Error (MSE) and the Peak Signal to Noise Ratio (PSNR) are the two error metrics used to compare image quality.The MSE represents the cumulative squared error between the compressed and the original image, whereas PSNR represents a measure of the peak. error. The lower the value of MSE, the lower the error.with the performance meterics such as PSNR and MSE, it was proved that fuzzy filter provides best results than the existing filters like wiener filter,median filter,order statistics filter.

For segmentation iterative thresholding can be used which will provide better results than the thresholding.Some times the thresholding technique can cause the loss of vital information. The comparison of segmentation is shown with the help of a table. From the table it is clear that iterative thresholding is best since its true positive value and false positive value is high. This is done by comparing the output of segmentation with the ground truth image.

The input CT image 1 of the lungs and the histogram of the image is shown in Fig2.

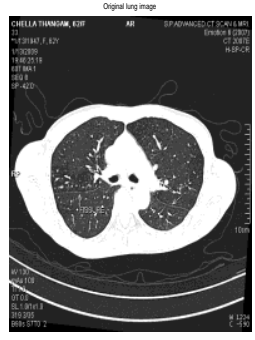

(a)

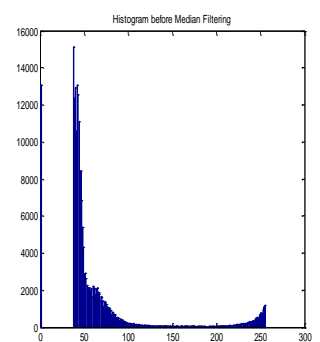

(b)

\section{Fig2.(a)input CT image of the lungs3,(b)Histogram of the input $\mathrm{CT}$ image}

The preprocessed image by $2 \mathrm{D}$ order statistics filtering and its histogram are shown below.The PSNR value of statistics filter is 16.6 .

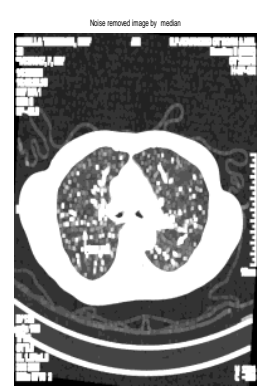

(a)

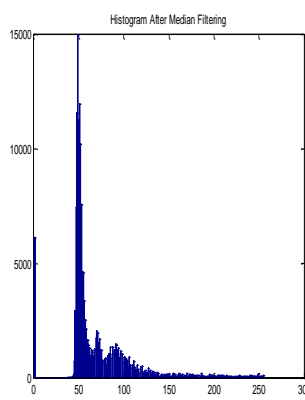

(b)
Fig3.(a)Preprocessed image by $2 D$ order statistics filtering,(b)Histogram after statistics filtering 
The preprocessed image by median filtering and its histogram are shown below.The PSNR value of statistics filter is 20 .

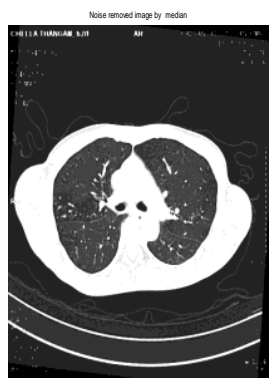

(a)

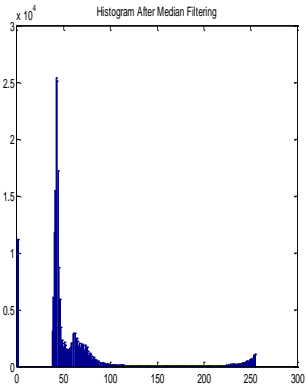

(b)
Fig4.(a)Preprocessed image by median filtering (b)Histogram after median filtering

The preprocessed image by wiener filtering and its histogram are shown below. The PSNR value of wiener filter is 31.894 .

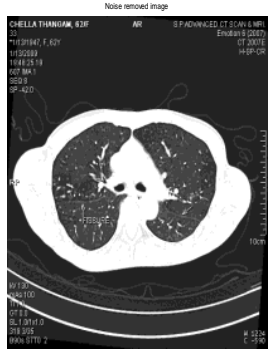

(a)

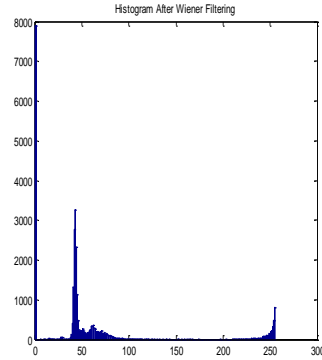

(b)
Fig5.(a)Preprocessed image by wiener filtering,(b) Histogram after wiener filtering

The preprocessed image by fuzzy filtering and its histogram are shown below.The PSNR value offuzzy filter is 34.57.

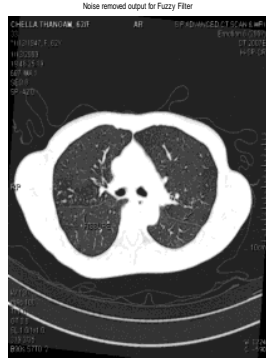

(a)

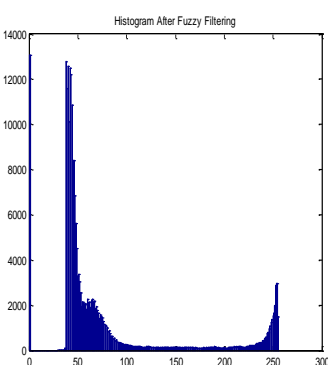

(b)
Fig6.(a)Preprocessed image by fuzzy filtering, (b) Histogram after fuzzy filtering

The bar graph of PSNR value of different types of filters are shown here. The fuzzy filter is having highest PSNR value.

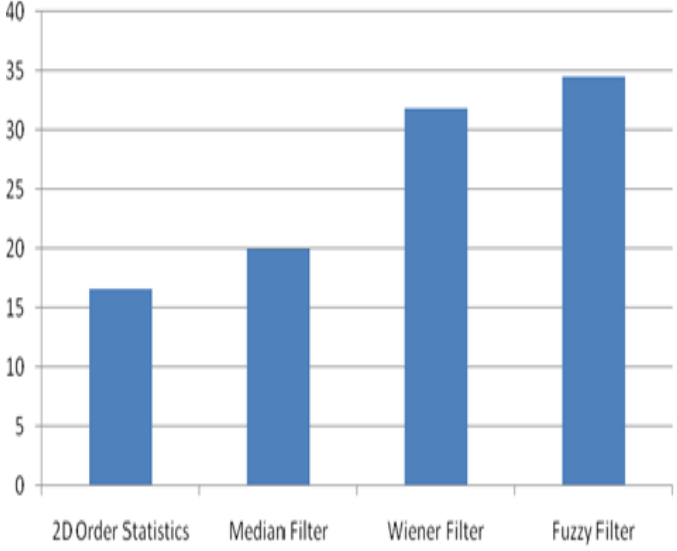

Fig 7: Bar Graph of PSNR value for different types of filter

The bar graph of MSE value of different types of filters are shown here. The fuzzy filter is having lowest MSE value.

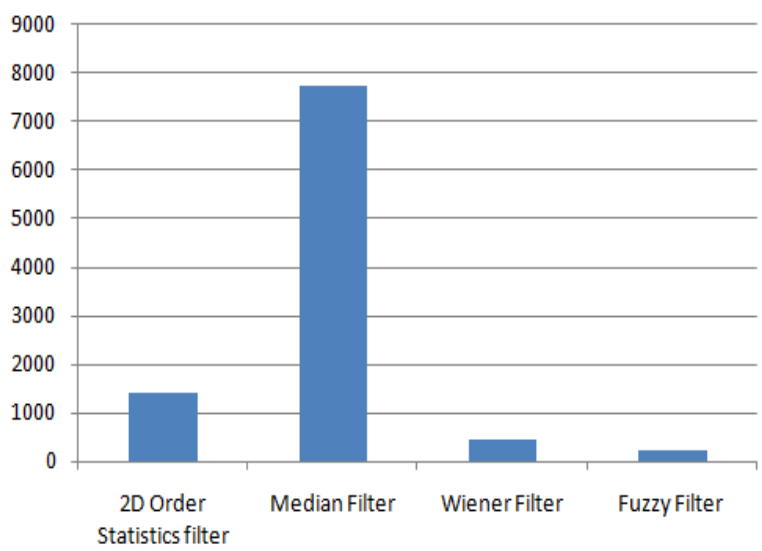

Fig 8: Bar Graph of MSE value for different types of filter

The segmentation output by iterative thresholding is shown here (Fig 9.a).The thresholding output and iterative thresholding output are compared with the ground truth image .Based on the comparison true positive value and false positive values are determined.

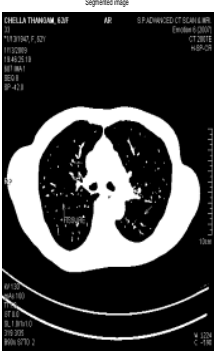

(a)

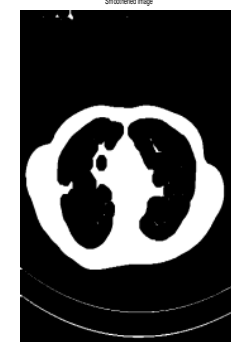

(b)
Fig9.(a)segmentationoutput,(b)Smoothened image 
True positive value represents the count that denotes the pixels (black) which are found in the ground truth image and segmented image. False positive value represents the count that denotes the pixels (White) which are found in the ground truth image and segmented image. From the results it is clear that iterative thresholding is better than thresholding.

Table 1: segmentation results

\begin{tabular}{|l|l|c|}
\hline Segmentation & $\begin{array}{l}\text { True } \\
\text { Positive } \\
\text { Value }\end{array}$ & $\begin{array}{l}\text { False Positive } \\
\text { value }\end{array}$ \\
\hline Thresholding & 34378 & 222403 \\
\hline Iterative Thresholding & 36329 & 222411 \\
\hline
\end{tabular}
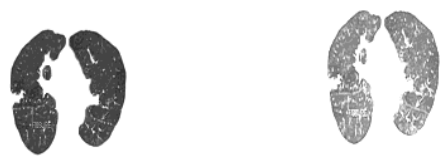

Fig10.(a)Fissure region sweeping,(b)Wavelet transform output

After the morphological operation the fissure region sweeping is done.The image is then subjected to wavelet transform. The wavelet transform output is as follows.

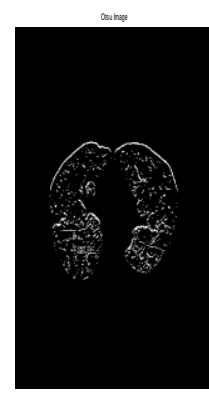

(a)

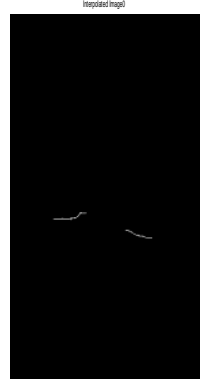

(b)

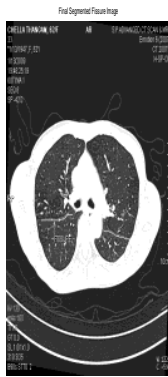

(c)
Fig11.(a)OtsusMethod,(b)Interpolation,Output(c) Extracted Lungs Image

The wavelet transform output is then subjected to otsus binarization technique.Finally the incomplete fissures are interpolated by linear interpolation technique.

The same steps were repeated for the real time CT image of other patients and satisfactory results were found.

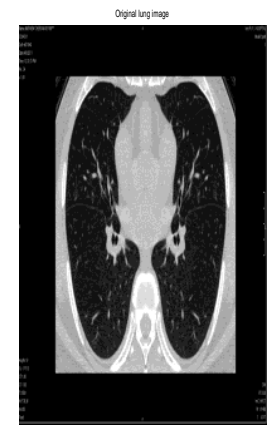

(a)

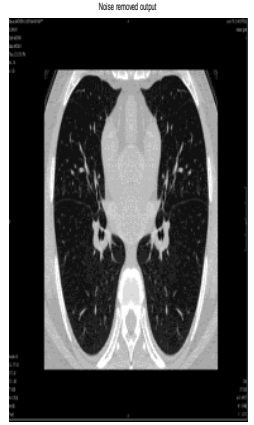

(b)
Fig12. (a) Input CT Image2,(b)Preprocessed Image

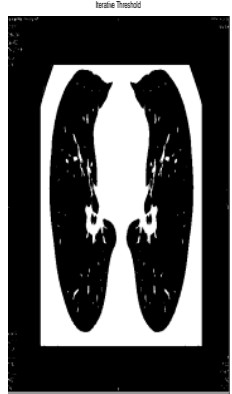

(a)

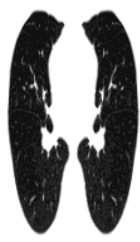

(b)
Fig 13(a) Iterative threshold output, (b) Extracted Lungs Array
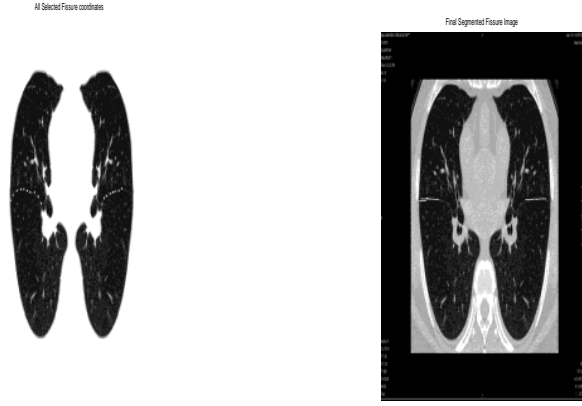

(a)

(b)

Fig 14.(a)Selecting Fissure coordinates,(b) Final Extracted Lungs

\section{CONCLUSION}

In conclusion, we developed a lobe segmentation algorithm for identifying lung lobes from CT images. Unlike previous segmentation methods using the bronchial tree, the lobe segmentation algorithm utilizes information from the bronchial tree without segmenting it to reduce the computation time and complexity.The fuzzy filter is used for preprocessing which is having high PSNR and low MSE In this paper, we present a segmentation algorithm using iterative thresholding and wavelet transform to segment lung lobes in isotropic CT images. The fissure regions are also 
analyzed for the treatment of lung cancer. Thus this work provides a preplanning for surgery.

\section{REFERENCES}

[1]. B. N. Raasch, E.W. Carsky, E. J. Lane, J. P. O’Callaghan, and E. R. Heitzman, "Radiographic anatomy of the interlobar fissures: A study of 100 specimens," Amer. J . Roentgenol., vol. 138, pp. 647-554, 1982

[2] M.Kass, A.Witkin, and D.Terzopoulos, "Snakes:Active contour models,'Int. J. Comput. Vis., vol. v1, pp. 321331, 1987.

[3]. R. M. Haralick, S. R. Stenberg, and X. Zhuang, "Image analysis using mathematical morphology," IEEE Trans. Pattern Anal. Mach. Intell., vol. 9, no. 4, pp. 532-550, Jul. 1987

[4]. M. Kubo, N. Niki, S. Nakagawa, K. Eguchi, M. Kaneko, N. Moriyama,H. Omatsu, R. Kakinuma, and N. Yamaguchi, "Extraction algorithm of pulmonary fissures from thin-section CT images based on linear feature detector method," IEEE Trans. Nucl. Sci., vol. 46, no. 6, pp. 2128-2133, Dec. 1999

[5]. M. Kubo, Y. Kawata, N. Niki, K. Eguchi, H. Ohmatsu, R. Kakinuma, M. Kaneko, M. Kusumoto, N. Moriyama, K. Mori, and H. Nishiyama, "Automatic extraction of pulmonary fissures from multidetector-row CT images," in Proc. IEEE Int. Conf. Imag. Process. (ICIP), pp. 10911094,2001

[6]. J.-M. Kuhnigk, H. Hahn, M. Hindennach, V. Dicken, S. Krass, and H.-O. Peitgen, "Lung lobe segmentation by anatomy-guided 3-D watershed transform," Proc. SPIE (Med. Imag.), vol. 5032, pp. 1482-1490,2003.
[7]. Takahiro Ohkawal, Syoji Kobashi', Katsuya Kondo', Yutaka Hatal, Tonioham Nakaiio','Tubular TissueBased Segmentation Of Lung Lobes From Chest Mdct Images", Cancer and Thoracic Surgery, Graduate School of Medicine, Okayama University, 2003.

[8]. Yoshinori itai,seji ishikawa,"Automatic segmentation of lung areas based on snakes and extraction of abnormal areas", 2005

[9]. .L.Zhang, E. A. Hoffman, and J. M. Reinhardt, "Atlasdriven lung lobe segmentation in volumetric X-ray CT images," IEEE Trans. Med. Imag., vol. 25, no. 1, pp. 116, Jan. 2006

[10].J.Wang, M. Betke, and J. P. Ko, "Pulmonary fissure segmentation on CT,"Med. Imag. Anal., vol. 10, pp $530-547,2006$

[11].American Cancer Society, Cancer Facts and Figures 2008. Atlanta, GA: American Cancer Society, 2008

[12].Jiantao Pu, Bin Zheng, Joseph K. Leader, Carl Fuhrman, Friedrich Knollmann, Amy Klym, and David Gur,"Pulmonary Lobe Segmentation in CT Examinations Using Implicit Surface Fitting"',IEEE transactions on medical imaging, vol. 28, no. 12, december 2009.

[13].Soumik Ukil,Joseph M Reinhardt,"Anatomy Guided Lung Lobe Segmentation In X-Ray Ct Images",Ieee Transactions On Medical Imaging,Vol 28,No2 Feburary 2009.

[14].z.Faizal khan,S.N Kumar,V.Kavitha," Efficient Algorithm to Enhance Lung Lobe Images using FuzzyFiltering",International Journal of Computer Applications(0975 - 8887) Volume 25- No.6, July 2011. 